\title{
Minimality of SRPT networks with resource sharing
}

\author{
ROBERT GIEROBA, ŁUKASZ KRUK \\ Department of Mathematics \\ Maria Curie-Skłodowska University \\ Pl. Marii Curie-Skłodowskiej 1, 20-031 Lublin \\ POLAND
}

\begin{abstract}
A general multi-resource network with users requiring service from a number of shared resources simultaneously is considered. It is demonstrated that the Shortest Remaining Processing Time (SRPT) service protocol minimizes, in a suitable sense, the system resource idleness with respect to customers with residual service times not greater than any threshold value on every network route. Our arguments are pathwise, with no assumptions on the model stochastic primitives and the network topology.
\end{abstract}

Key-Words: Queuing, resource sharing, SRPT, minimality, residual file sizes, partial ordering, service protocols

Received: November 21, 2020. Revised: February 28, 2021. Accepted: March 18, 2021. Published: April 1, 2021.

\section{Introduction}

The Shortest Remaining Processing Time (SRPT) service discipline assigns preemptive priority to the task with the smallest residual service time. It is well known in queueing theory for minimizing the mean sojourn time (Schrage and Miller [1]) and the queue length in a single-server system (Schrage [2]). A summary of early work on SRPT may be found in a survey paper by Schreiber [3]. Some of more recent studies of this protocol discuss advisability of implementing it from the point of view of fairness (e.g., Wierman and Harchol-Balter [4]) or tail behavior (Núñez-Queija [5], Nuyens and Zwart [6]). Heavy traffic limit of the invariant queue length distributions in an M/G/1 SRPT system was developed by Pavlov [7] and Pechinkin [8]. There is also a growing body of literature concerning functional limit theorems for SRPT queues, see a recent paper by Banerjee, Budhiraja and Puha [9] and the references given there. So far, vast majority of studies in this area, including the ones cited above, has been concentrated on the performance of single-server systems. Consequently, relatively little is known about the performance of queueing networks using the SRPT protocol.

Among many network types in operations research literature, there are resource sharing networks, also called bandwidth sharing models or flow level models. These systems were introduced by Massoulié and Roberts [10, 11] in order to study problems of congestion control of the Internet traffic. In such models flows, corresponding to continuous transmissions of elastic documents, are being transferred on typespecific routes, needing simultaneous service at all the resources on their paths. Few results on the implementation of the SRPT protocol in such systems are available in the existing literature. Verloop, Borst and Núñez-Queija [12] demonstrated that strictly subcritical networks with resource sharing and linear topology, working under SRPT, as well as the shortest expected remaining processing time (SERPT) and the least attained service (LAS) scheduling, can be unstable. This suggested applying SRPT only locally, as an intra-route discipline, on the top of another policy allocating bandwidth to routes in a resource sharing network, see Aalto and Ayesta [13]. Chojecki and Kruk [14] constructed a related example of a strictly subcritical multiclass queueing network which is unstable with the SRPT, SERPT and the shortest job first (SJF) scheduling policies. Note that in multiclass queueing networks, customers visit different stations along their routes in succession, so these systems differ notably from the ones considered here.

In this paper, we propose a new performance evaluation methodology for size-based policies in general resource sharing networks. To this end, we introduce a suitable idleness (equivalently, transmission time) based optimality criterion, described below. For every $i$ from the set $\mathbf{I}$ of the network routes and any times $t, s \geq 0$, we define $Y_{i}(t, s)$ as the cumulative idleness by time $t$ with regard to transferring flows having residual transmission times (evaluated during their processing) not greater than $s$. We compare the effects of implementing different service disciplines for a given network by comparing the corresponding vector-valued functions $Y=\left(Y_{i}\right)_{i \in \mathbf{I}}$ with respect to the pointwise functional inequality. This introduces 
a partial ordering which is, in general, not linear. A minimizing discipline with respect to this relation (if it exists) is called minimal. Note that some pairs of service disciplines may not be comparable in this sense, so in general more than one minimal protocol may exist. However, in the absence of any distributional assumptions on the underlying stochastic primitives, we show that the SRPT resource sharing discipline is minimal. A partial converse to this finding is the fact that in a minimal network with resource sharing, flows on each route are transmitted according to the SRPT discipline. Consequently, a single-server, single customer class queue is minimal if and only if it is working under the SRPT protocol.

Our minimality notion is a counterpart of pathwise minimality for real-time resource sharing networks introduced by Kruk [15], where lead times of the flows, rather than their residual service times, correspond to the second coordinate of the functions $\left(Y_{i}\right)_{i \in \mathbf{I}}$. Related minimality criteria for real-time networks with resource sharing may also be found in Kruk [16]. For real-time systems, the Earliest Deadline First (EDF), rather than SRPT, is optimal. Although our results are qualitatively similar to those from [15], their proofs turn out to be notably different. Indeed, the flow's lead time always decreases at the unit rate, regardless of its transmission, so, in particular, the relative priorities of flows in the EDF system are always the same. In contrast, the residual transfer times of the flows decrease at their transmission rates, which vary over time and are different for different flows. Hence, in SRPT systems, tasks may change their relative priorities over time, making the corresponding arguments more involved.

To our knowledge, our study is the first one in which SRPT resource sharing networks with arbitrary topology are investigated. In contrast to previous research concerning the SRPT protocol (with the notable exception of the classic result of Schrage [2]), our arguments are pathwise, and hence they are not constrained to any particular class of interarrival and/or service time distributions. Moreover, we present an advantage of implementing the SRPT protocol in this context, namely its minimality in the sense discussed above, while the previous study [12] exhibited its disadvantage (instability). Consequently, although our work is theoretical in nature, it may be regarded as an encouragement for practitioners to use SRPT as a transmission protocol whenever possible (i.e., when the corresponding document sizes are known in advance), at least as an intra-route discipline.

Finally, let us mention that there are numerous practice-oriented papers on the Internet traffic available in the computer science and engineering literature. Some recent studies of this kind can be found, e.g., in [17] and [18].

\section{Notation}

Let $\mathbb{R}$ denote the set of real numbers and let $\mathbb{R}_{+}=$ $[0,+\infty)$. For $a, b \in \mathbb{R}$, we write $a \vee b(a \wedge b)$ for the maximum (minimum) of $a$ and $b$, and $a^{+}$for $a \vee 0$. Vector inequalities should be understood componentwise: for $a, b \in \mathbb{R}^{n}, a=\left(a_{1}, . . a_{n}\right), b=\left(b_{1}, \ldots, b_{n}\right)$, $a \leq b$ if and only if $a_{i} \leq b_{i}$ for $i=1, \ldots, n$. Functional inequalities should be understood in a pointwise sense, i.e., for $f, g: A \rightarrow \mathbb{R}^{n}$, we write $f \leq g$ if and only if $f(x) \leq g(x)$ for all $x \in A$. A sum over the empty set of indices will be regarded as zero.

The $\sigma$-field of the Borel subsets of $\mathbb{R}_{+}$will be denoted by $\mathcal{B}\left(\mathbb{R}_{+}\right)$. We write $\mathbb{I}_{B}$ for the indicator of a set $B \in \mathcal{B}\left(\mathbb{R}_{+}\right)$. For a function $f(x, y)$ of two variables, let $d_{x} f(x, y)$ denote the differential of $f(x, y)$ with respect to $x$, i.e., $d g_{y}(x)$, where $g_{y}(x)=f(x, y)$ is a function of $x$ depending on a parameter $y$.

Let $\mathbf{M}$ be the set of nonnegative, finite measures on $\mathcal{B}\left(\mathbb{R}_{+}\right)$. When $\mu \in \mathbf{M}$ and $a, b \in \mathbb{R}_{+} \cup\{\infty\}$, we write $\mu(a, b), \mu[a, b), \mu(a, b], \mu[a, b]$ instead of $\mu((a, b)), \mu([a, b)), \mu((a, b]), \mu([a, b])$ respectively. We also often write $\mu(x)$ instead of $\mu(\{x\})$ to denote the measure $\mu$ of a set consisting of a single element $x$. For $\mu \in \mathbf{M}$, let $L_{\mu}=\sup \left\{x \in \mathbb{R}_{+}: \mu[0, x)=0\right\}$. Thus, we have $\mu\left(\mathbb{R}_{+}\right)=0$ if and only if $L_{\mu}=\infty$. The family $\mathbf{M}$ is equipped with the weak topology, which makes it a Polish space (see Prohorov [19]). We denote the measure in $\mathbf{M}$ that puts the unit of mass at a point $x \in \mathbb{R}$ by $\delta_{x}$.

Random processes analyzed in this article have sample paths that are right continuous with finite left limits (r.c.l.1.). For a Polish space $\mathcal{S}, \mathbf{D}([0, \infty), \mathcal{S})$ is the space of r.c.l.1. functions from $[0, \infty)$ into $\mathcal{S}$. For $x \in \mathbf{D}\left([0, \infty), \mathbb{R}^{n}\right)$ and $t>0$, let $\triangle x(t)=$ $x(t)-x(t-)$, where $x(t-)=\lim _{s \rightarrow t^{-}} x(s)$.

\section{Stochastic model}

\subsection{Network description}

We consider a network possessing a finite set of routes, indexed by $i=1, \ldots, I$, and a finite number of resources (nodes), indexed by $j=1, \ldots, J$. Every route may be formally regarded as a nonempty subset of $\mathbf{J}=\{1, \ldots, J\}$, namely the set of resources used by this route. We define the $J \times I$ incidence matrix $A=\left[a_{j i}\right]$, letting $a_{j i}=1$ if resource $j$ is used by route $i$ and $a_{j i}=0$ otherwise. Let $\mathbf{I}=\{1, \ldots, I\}$. In terms of $A$, the set of resources used by route $i$ may be defined as $\mathcal{R}(i)=\left\{j \in \mathbf{J}: a_{j i}=1\right\}$ and the set of routes using the resource $j$ is described as $\mathcal{F}(j)=\left\{i \in \mathbf{I}: a_{j i}=1\right\}$.

By a flow on route $i$ we mean a continuous transmission of a file through the resources used by this 
route. As it is standard in the resource sharing network literature, we assume that a flow takes simultaneous possession of all the resources on its route during the transmission. For convenience, we also assume that all the network resources have the same maximal capacity (i.e., service rate). Without loss of generality, this common transmission rate may be taken to be one (if this is not the case, we can perform an obvious time rescaling).

Let $(\Omega, \mathcal{A}, \mathbf{P})$ be the underlying probability space. The initial condition for the network model consists of the nonnegative, integer-valued random variables $Q_{i}(0), i \in \mathbf{I}$, representing the numbers of initial flows on each route at time zero, and the strictly positive random initial flow sizes $\tilde{v}_{i, k}$, where $i \in \mathbf{I}$, $k=1, \ldots, Q_{i}(0)$. The initial flow with service time $\tilde{v}_{i, k}$ will be referred to as flow $k$ on route $i$. Put $Q(0)=\left(Q_{1}(0), \ldots, Q_{I}(0)\right)$.

For $i \in \mathbf{I}$ and $t \geq 0$, let $N_{i}(t)$ denote the number of flows arriving to the $i$-th route in the time interval $(0, t]$. The $k$-th arrival modelled by the process $N_{i}(\cdot)$ will be referred to as flow $Q_{i}(0)+k$ on route $i$. Its arrival time equals $U_{i, k}=\inf \left\{t \geq 0: N_{i}(t) \geq k\right\}$. For $i \in \mathbf{I}$ and $t \geq 0$, let $A_{i}(t)=Q_{i}(0)+N_{i}(t)$.

For $i \in \mathbf{I}, k \geq 1$, let $v_{i, k}$ denote the initial file size of the $Q_{i}(0)+\bar{k}$-th flow on route $i$. By assumption, all the random variables $v_{i, k}$ are greater than zero.

\subsection{Basic performance processes}

For $i \in \mathbf{I}, t \geq 0$ and $k \leq A_{i}(t)$, let $w_{i, k}(t)$ denote the residual file size (transmission time) of flow $k$ on route $i$ at time $t$. Note that $w_{i, k}(\cdot)$ decreases during the transmission of the flow $k$ on route $i$ according to the transmission rate assigned to this flow and it is constant otherwise. For convenience, we denote by $w_{i, k}^{0}$ the initial file sizes, i.e., $w_{i, k}^{0}=\tilde{v}_{i, k}$ for initial flows $k \leq Q_{i}(0)$ and $w_{i, k}^{0}=v_{i, k-Q_{i}(0)}$ for incoming flows $k>Q_{i}(0)$.

The stochastic primitives described above determine the measure-valued arrival process

$$
\mathcal{A}_{i}(t)=\sum_{k=1}^{Q_{i}(0)} \delta_{\tilde{v}_{i, k}}+\sum_{k=1}^{N_{i}(t)} \delta_{v_{i, k}}, \quad i \in \mathbf{I}, \quad t \geq 0 .
$$

For $i \in \mathbf{I}$ and $t \geq 0$, let us define the measure-valued state descriptor for route $i$ as

$$
\mathcal{Q}_{i}(t)=\sum_{k=1}^{A_{i}(t)} \mathbb{I}_{(0, \infty)}\left(w_{i, k}(t)\right) \delta_{w_{i, k}(t)} .
$$

The random measure $\mathcal{Q}_{i}(t)$ has the unit mass at the residual transmission time of each flow on route $i$ at time $t$. The quantity $Q_{i}(t)=\mathcal{Q}_{i}(t)\left(\mathbb{R}_{+}\right)$denotes the number of flows on the route $i \in \mathbf{I}$ at time $t$. Let $Q(t)=\left(Q_{1}(t), \ldots, Q_{I}(t)\right)$.
We define the current shortest remaining processing time process for route $i$ by the formula $C_{i}(t)=$ $L_{\mathcal{Q}_{i}(t)}, t \geq 0$.

\subsection{SRPT discipline}

In what follows, we will study the SRPT service protocol, dynamically allocating bandwidth to flows with the shortest residual transmission times. We assume preempt-resume and no overhead. Below, we shall define this protocol carefully, introducing notation which will be used in the proofs of our results.

Let $t \geq 0$ be such that $Q(t) \neq 0$ and let $i_{0} \in \mathbf{I}$, $k_{0} \leq A_{i_{0}}(t)$ be such that $w_{i_{0}, k_{0}}(t)$ is the minimum of the residual transmission times of the flows present in the network at time $t$. Throughout this paper, we assume an arbitrary tie-breaking rule. The flow $k_{0}$ on route $i_{0}$ is selected for transmission at time $t$. Define $\mathbf{J}_{1}=\mathbf{J} \backslash \mathcal{R}\left(i_{0}\right), \mathbf{I}_{1}=\left\{i \in \mathbf{I}: \mathcal{R}(i) \subseteq \mathbf{J}_{1}\right\}$. If $\sum_{i \in \mathbf{I}_{1}} Q_{i}(t)=0$ (for example, if $\mathbf{I}_{1}=\emptyset$ ), then the assignment of flows for transmission at time $t$ is finished. In the opposite case, let $i_{1} \in \mathbf{I}_{1}, k_{1} \leq$ $A_{i_{1}}(t)$ be such that $w_{i_{1}, k_{1}}(t)$ is the minimum of the residual transmission times of the flows which are on routes belonging to $\mathbf{I}_{1}$ at time $t$. We choose the flow $k_{1}$ on route $i_{1}$ for transmission at time $t$. Take $\mathbf{J}_{2}=\mathbf{J}_{1} \backslash \mathcal{R}\left(i_{1}\right), \mathbf{I}_{2}=\left\{i \in \mathbf{I}: \mathcal{R}(i) \subseteq \mathbf{J}_{2}\right\}$. If $\sum_{i \in \mathbf{I}_{2}} Q_{i}(t)=0$ (for example, if $\mathbf{I}_{2}=\bar{\emptyset}$ ), we are done, otherwise we continue in this way until, at some step $n$, we get $\sum_{i \in \mathbf{I}_{n}} Q_{i}(t)=0$, ending the selection procedure at time $t$. This assignment is used until either one of the current transmissions is finished, or a new flow arrives at the network, when, according to the same algorithm, rescheduling can occur.

We will also analyze protocols allocating transmission rates to routes differently than SRPT, but scheduling flows on any route according to the SRPT policy, that is, prioritizing the files with the smallest residual transmission times. For example, we can consider a resource sharing network having fixed route priorities, in which files on each route are transmitted in the SRPT order.

\subsection{Network equations}

For $i \in \mathbf{I}$ and $t, s \geq 0$, define

$$
\begin{gathered}
E_{i}(t, s)=\sum_{k=1}^{N_{i}(t)} \delta_{v_{i, k}}(0, s]=\mathcal{A}_{i}(t)(0, s]-\mathcal{Q}_{i}(0)(0, s], \\
Z_{i}(t, s)=\mathcal{Q}_{i}(t)(0, s] .
\end{gathered}
$$

Note that $N_{i}(t)=\lim _{s \rightarrow \infty} E_{i}(t, s), Q_{i}(t)=$ $\lim _{s \rightarrow \infty} Z_{i}(t, s)$. Let $E(t, s)=\left(E_{i}(t, s)\right)_{i \in \mathbf{I}}$, $Z(t, s)=\left(Z_{i}(t, s)\right)_{i \in \mathbf{I}}$. Let the vectors $D(t)=$ $\left(D_{i}(t)\right)_{i \in \mathbf{I}}, T(t, s)=\left(T_{i}(t, s)\right)_{i \in \mathbf{I}}$ denote the number of departures (that is, service completions) and the cumulative transmission time by time $t$ corresponding 
to every route $i$ of flows with residual transmission times (evaluated at the times of their processing) not greater than $s$. Let $Y_{i}(t, s)=t-T_{i}(t, s), i \in \mathbf{I}$, denote the cumulative idleness by time $t$ with regard to the service of of flows on route $i$ with residual transmission times (evaluated at the times of their processing) not greater than $s$ and let $Y(t, s)=\left(Y_{i}(t, s)\right)_{i \in \mathbf{I}}$. For $i \in \mathbf{I}, t, s \geq 0$, let $P(t, s)$ denote the number of flows arriving to route $i$ by time $t$ (including the initial ones) with transmission times initially greater than $s$, but partially processed so that their residual transmission times at time $t$ are not greater than $s$. Let us stress that the functions $P_{i}$ depend on the network discipline or, more precisely, on the functions $T_{i}$ corresponding to this protocol. This fact is not reflected in the notation.

For $t \geq 0$ and $s \geq 0$, define

$$
\mathfrak{X}(t, s)=(Z(t, s), D(t), T(t, s), Y(t, s)) .
$$

Note that all the coordinate processes of $\mathfrak{X}$ are nonnegative. The processes $D(\cdot), T(\cdot, s), Y(\cdot, s)$ are nondecreasing, with $D(0)=T(0, s)=Y(0, s)=0$ for $s \geq 0$. Also, the functions $Z(t, \cdot)$ and the increments $T(\tilde{t}, \cdot)-T(t, \cdot), Y(t, \cdot)-Y(\tilde{t}, \cdot)$ are nondecreasing for every $\tilde{t} \geq t \geq 0$. The following network equations

$$
\begin{aligned}
& Z(t, s)=Z(0, s)+E(t, s)+P(t, s)-D(t), \\
& D_{i}(t)=P_{i}(t, 0), i \in \mathbf{I}, \\
& T_{i}(t, s)+Y_{i}(t, s)=t, i \in \mathbf{I}, \\
& \sum_{i \in \mathcal{F}(j)}\left(T_{i}(\tilde{t}, s)-T_{i}(t, s)\right) \leq \tilde{t}-t, j \in \mathbf{J},
\end{aligned}
$$

hold for $\tilde{t} \geq t \geq 0, s \geq 0$. The equation (5) above reflects the capacity constraints of the network resources. We also impose the following additional condition

$$
\int_{0}^{t} \mathbb{I}_{\left[Z_{i}(u, s)=0\right]} d_{u} T_{i}(u, s)=0, \quad i \in \mathbf{I},
$$

valid for all $t, s \geq 0$. It expresses a natural requirement that flows that are not present in the system cannot be transmitted.

The equations (2)-(6) hold for the performance processes of the form (11) describing the implemantation of any reasonable protocol, including SRPT, in the network under consideration. Hence, in the next sections, we will always assume their validity.

\section{An auxiliary result}

The following proposition gives us a useful one-toone correspondence between the performance processes $Z_{i}$ and $Y_{i}$ (or, equivalently, $T_{i}$ ), $i \in \mathbf{I}$.
Proposition 1. Consider two performance processes: $\mathfrak{X}(t, s)$ given by (11) and $\mathfrak{X}^{\prime}(t, s)=$ $\left(Z^{\prime}(t, s), D^{\prime}(t), T^{\prime}(t, s), Y^{\prime}(t, s)\right), t \geq 0, s \geq 0$ satisfying the same conditions as $\mathfrak{X}(t, s)$. By $E_{i}^{\prime}$, $i \in \mathbf{I}$ we denote the random field, analogous to $E_{i}$, corresponding to the process $\mathfrak{X}^{\prime}$. Let $i \in \mathbf{I}$, $T \in(0,+\infty)$. If $Z_{i}(0, \cdot)=Z_{i}^{\prime}(0, \cdot)$ and $E_{i}(t, \cdot) \equiv$ $E_{i}^{\prime}(t, \cdot), Y_{i}(t, \cdot) \equiv Y_{i}^{\prime}(t, \cdot)$ for every $t \in[0, T]$, then $Z_{i}(t, \cdot) \equiv Z_{i}^{\prime}(t, \cdot)$ for every $t \in[0, T]$.

Proof. Recall that for each $t \geq 0, Z_{i}(t, \cdot)$ is a distribution function of a finite measure $\mathcal{Q}_{i}(t)$ with atoms of integer sizes. Denote by $\mathcal{Q}_{i}^{\prime}(t)$ the measure determined by $Z_{i}^{\prime}(t, \cdot)$. Let

$$
t_{0}=\sup \left\{\bar{t} \geq 0: Z_{i}^{\prime}(t, \cdot) \equiv Z_{i}(t, \cdot) \forall t \in[0, \bar{t}]\right\} .
$$

Observe that if $t_{0}>0$, then $Z_{i}\left(t_{0}, \cdot\right)=Z_{i}^{\prime}\left(t_{0}, \cdot\right)$. Indeed, $\mathcal{Q}_{i}\left(t_{0}-\right)=\mathcal{Q}_{i}^{\prime}\left(t_{0}-\right)$ implies in particular that $\mathcal{Q}_{i}\left(t_{0}-\right)(0)=\mathcal{Q}_{i}^{\prime}\left(t_{0}-\right)(0)$. Since $E_{i}(t, \cdot) \equiv E_{i}^{\prime}(t, \cdot)$ for $t \in[0, T], N_{i} \equiv N_{i}^{\prime}$ on $[0, T]$, where $N_{i}^{\prime}(t)=$ $\lim _{s \rightarrow \infty} E_{i}^{\prime}(t, s)$. Moreover, the random measure

$$
\mathcal{A}_{i}^{\prime}(t)=\sum_{k=1}^{Q_{i}^{\prime}(0)} \delta_{\tilde{v}_{i, k}^{\prime}}+\sum_{k=1}^{N_{i}^{\prime}(t)} \delta_{v_{i, k}^{\prime}},
$$

where $\tilde{v}_{i, k}^{\prime}$ and $v_{i, k}^{\prime}$ are defined analogously as $\tilde{v}_{i, k}$ and $v_{i, k}$, is equal to $\mathcal{A}_{i}(t)$ for $t \in[0, T]$. Flows whose residual processing times reach zero at time $t_{0}$ leave the system at this time, so

$$
\begin{aligned}
\mathcal{Q}_{i}\left(t_{0}\right) & =\mathcal{Q}_{i}\left(t_{0}-\right)-\mathcal{Q}_{i}\left(t_{0}-\right)(0) \delta_{0} \\
& +\mathcal{A}_{i}\left(t_{0}\right)-\mathcal{A}_{i}\left(t_{0}-\right)= \\
& =\mathcal{Q}_{i}^{\prime}\left(t_{0}-\right)-\mathcal{Q}_{i}^{\prime}\left(t_{0}-\right)(0) \delta_{0} \\
& +\mathcal{A}_{i}^{\prime}\left(t_{0}\right)-\mathcal{A}_{i}^{\prime}\left(t_{0}-\right)=\mathcal{Q}_{i}^{\prime}\left(t_{0}\right),
\end{aligned}
$$

and hence $Z_{i}\left(t_{0}, \cdot\right) \equiv Z_{i}^{\prime}\left(t_{0}, \cdot\right)$.

If $t_{0}=T$, the proof is finished, so assume that $t_{0}<T$. Let $\varepsilon$ be the minimal distance between the atoms of the measure $\mathcal{Q}_{i}\left(t_{0}\right)=\mathcal{Q}_{i}^{\prime}\left(t_{0}\right)$ (i.e. the residual transmission times of the flows present in both systems at time $\left.t_{0}\right)$. Put

$$
\begin{array}{r}
t_{1}=\inf \left\{t_{0}<t \leq T: \triangle N(t) \neq 0 \text { or } \triangle Q(t) \neq 0\right. \\
\text { or } \left.\triangle Q^{\prime}(t) \neq 0\right\},
\end{array}
$$

and let $t \in\left(t_{0}, t_{1}\right), t-t_{0} \leq \varepsilon / 2$, be such that $Z_{i}(t, \cdot) \neq Z_{i}^{\prime}(t, \cdot)$. By the definition of $t_{1}$ there are no external arrivals and no departures in both systems under consideration in the time interval $\left(t_{0}, t_{1}\right)$. Let

$$
s_{*}=\sup \left\{\bar{s} \geq 0: Z_{i}^{\prime}(t, s) \equiv Z_{i}(t, s) \forall s \in[0, \bar{s}]\right\} .
$$

By definition, $Z_{i}\left(t, s_{*}\right) \neq Z_{i}^{\prime}\left(t, s_{*}\right)$, otherwise we would have $Z_{i}(t, s)=Z_{i}^{\prime}(t, s)$ for $s \in\left[0, s_{*}+\varepsilon\right)$ since the functions $Z_{i}(t, \cdot), Z_{i}^{\prime}(t, \cdot)$ are r.c.l.1. and 
piecewise constant (as distribution functions of purely atomic measures with finitely many atoms), but this would contradict the definition of $s_{*}$. We can assume that $Z_{i}\left(t, s_{*}\right)<Z_{i}^{\prime}\left(t, s_{*}\right)$. This means that in the system $\mathfrak{X}^{\prime}$ there is at least one flow with residual transmission time $s_{*}$ more than in the system $\mathfrak{X}$ and its residual transmission time in the system $\mathfrak{X}$ is greater than $s_{*}$.

Let $s^{*}$ be the smallest atom of $\mathcal{Q}_{i}(t)+\mathcal{Q}_{i}^{\prime}(t)$ greater than $s_{*}$, i.e.

$$
s^{*}=\min \left\{s>s_{*}:\left(\mathcal{Q}_{i}(t)+\mathcal{Q}_{i}^{\prime}(t)\right)(s)>0\right\} .
$$

For any $k \leq A_{i}(t)$ we have $0 \leq w_{i, k}(t)-w_{i, k}\left(t_{0}\right) \leq$ $t-t_{0} \leq \varepsilon / 2$. Therefore, if $w_{i, k}(t)=s_{*}$, then $w_{i, k}\left(t_{0}\right)=S_{*}$, where $S_{*}$ is the unique (by the definition of $\varepsilon$ ) atom of $\mathcal{Q}_{i}\left(t_{0}\right)=\mathcal{Q}_{i}^{\prime}\left(t_{0}\right)$ in the interval $\left[s_{*}, s_{*}+\varepsilon / 2\right]$. Furthermore, by the definition of $\varepsilon$, if $w_{i, k}\left(t_{0}\right) \neq S_{*}$, then either $w_{i, k}\left(t_{0}\right) \leq S_{*}-\varepsilon \leq$ $s_{*}-\varepsilon / 2$ or $w_{i, k}\left(t_{0}\right) \geq S_{*}+\varepsilon \geq s_{*}+\varepsilon$. Analogous observations also hold for the system $\mathfrak{X}^{\prime}$.

Observe that $s^{*} \leq S_{*}$. The number of flows with residual transmission times $s_{*}$ at time $t$ is greater in the system $\mathfrak{X}^{\prime}$, while the same number of flows with residual transmission times $S_{*}$ at time $t_{0}$ is present in both systems by the definition of $t_{0}$. Therefore, there must exist at least one flow in the system $\mathfrak{X}$ with residual transmission time $S_{*}$ at $t_{0}$, the residual transmission time of which is greater than $s_{*}$ at time $t$. This residual transmission time cannot be greater than $S_{*}$, because it cannot increase and it cannot be smaller than $s^{*}$ by the definition of $s^{*}$. This implies in particular that $s^{*}-s_{*} \leq \varepsilon / 2$. Moreover, for $u \in\left[t_{0}, t\right]$, if $w_{i, k}(u) \in\left[s_{*}, s^{*}\right]$, then $w_{i, k}\left(t_{0}\right)=S_{*}$, since $s^{*} \leq s_{*}+\varepsilon / 2$ and $S_{*}$ is the unique atom of $\mathcal{Q}_{i}\left(t_{0}\right)$ in the interval $\left[s_{*}, s_{*}+\varepsilon / 2\right]$. Similarly, if $w_{i, k}^{\prime}(u) \in\left[s_{*}, s^{*}\right]$, then $w_{i, k}^{\prime}\left(t_{0}\right)=S_{*}$. Thus

$$
\begin{aligned}
& \left(T_{i}\left(t, s^{*}\right)-T_{i}\left(t, s_{*}\right)\right)-\left(T_{i}\left(t_{0}, s^{*}\right)-T_{i}\left(t_{0}, s_{*}\right)\right) \\
= & \left(s^{*}-s_{*}\right)\left[\mathcal{Q}_{i}(t)\left(s_{*}\right)+\sum_{S_{*}-\varepsilon / 2 \leq s<s_{*}} \mathcal{Q}_{i}(t)(s)\right] .
\end{aligned}
$$

Indeed, the left-hand side of this equality is the amount of work done on route $i$ in the time interval $\left[t_{0}, t\right]$ to process flows with residual processing times in $\left(s_{*}, s^{*}\right]$. By the definitions of $s_{*}, s^{*}$ and $S_{*}$ those flows have $w_{i, k}(t) \leq s_{*}$ and $w_{i, k}\left(t_{0}\right)=S_{*}$ at the times of their processing in this time interval. This work changed their residual transmission times from $s^{*}$ to $s_{*}$, therefore the amount of work is the righthand side of the equality. The last sum represents tasks which had $w_{i, k}\left(t_{0}\right)=S_{*}$ and ended up with $w_{i, k}(t)<s_{*}$, if such tasks exist. Such tasks are identical in both systems under consideration. Similarly, we have

$$
\begin{aligned}
& \left(T_{i}^{\prime}\left(t, s^{*}\right)-T_{i}^{\prime}\left(t, s_{*}\right)\right)-\left(T_{i}^{\prime}\left(t_{0}, s^{*}\right)-T_{i}^{\prime}\left(t_{0}, s_{*}\right)\right) \\
= & \left(s^{*}-s_{*}\right)\left[\mathcal{Q}_{i}^{\prime}(t)\left(s_{*}\right)+\sum_{S_{*}-\varepsilon / 2 \leq s<s_{*}} \mathcal{Q}_{i}^{\prime}(t)(s)\right] .
\end{aligned}
$$

This means that if $T_{i}(t, \cdot) \equiv T_{i}^{\prime}(t, \cdot)$ for all $t \in[0, T]$, then $\mathcal{Q}_{i}(t)\left(s_{*}\right)=\mathcal{Q}_{i}^{\prime}(t)\left(s_{*}\right)$, which in turn contradicts the definition of $s_{*}$.

Corollary 1. Under the assumptions of Proposition 1. we have $D_{i}(t, \cdot) \equiv D_{i}^{\prime}(t, \cdot)$ for $t \in[0, T], i \in \mathbf{I}$.

Indeed, $D_{i}$ and $D_{i}^{\prime}$ are piecewise constant functions and for $t \in[0, T]$,

$$
\begin{aligned}
\triangle D_{i}(t) & =\triangle N_{i}(t)-\triangle Q_{i}(t)=\triangle N_{i}^{\prime}(t)-\triangle Q_{i}^{\prime}(t)= \\
& =\triangle D_{i}^{\prime}(t) .
\end{aligned}
$$

\section{Minimality}

Recall that the equations (2)-(6) are of a very general nature and they hold for networks equipped with many different protocols. In particular, they do not impose any lower bounds on the transmission rates, allowing for arbitrarily large system idleness. In fact, even an idle network with $Z(t, s)=Z(0, s)+E(t, s)$, $Y_{i}(t, s)=t$ and $D(t)=T(t, s)=P(t, s)=0$ for each $i \in \mathbf{I}, t, s \geq 0$, satisfies (2)-(6), as well as the assumptions listed below (11). We will now introduce a concept of minimality implying the transmition of flows on every given route in the SRPT order (see Theorem 2, to follow) and enforcing a counterpart of non-idleness (known from multiclass queueing networks) suitable for networks with resource sharing.

Definition 1. Suppose that two performance processes of the form (11) for resource sharing networks, namely, $\mathfrak{X}^{(1)}=\left(Z^{(1)}, D^{(1)}, T^{(1)}, Y^{(1)}\right)$ and $\mathfrak{X}^{(2)}=$ $\left(Z^{(2)}, D^{(2)}, T^{(2)}, Y^{(2)}\right)$, satisfy (2)-(6), as well as the remaining requirements listed below (11). We assume that they have common stochastic primitives (i.e., the initial condition $Z^{(1)}(0, \cdot) \equiv Z^{(2)}(0, \cdot)$ and the external arrival function $E$ ), and possess the same incidence matrix $A$. By $\mathfrak{X}^{(1)} \preceq \mathfrak{X}^{(2)}$ we denote the relation $Y^{(1)}(\omega) \leq Y^{(2)}(\omega)$ (which is equivalent to $\left.T^{(1)}(\omega) \geq T^{(2)}(\omega)\right)$ holding for all $\omega \in \Omega$.

Let us remark that by the notational conventions from Section 2, the above condition $Y^{(1)}(\omega) \leq$ $Y^{(2)}(\omega)$ means that $Y_{i}^{(1)}(t, s)(\omega) \leq Y_{i}^{(2)}(t, s)(\omega)$ for all $i \in \mathbf{I}$ and $t, s \geq 0$.

Definition 2. Let $\mathfrak{X}$ be a performance process, satisfying (11)- (6) and the nonnegativity and monotonicity requirements listed below (1). We say that $\mathfrak{X}$ is minimal if for any process $\mathfrak{X}^{\prime}$, the inequality $\mathfrak{X}^{\prime} \preceq \mathfrak{X}$ implies that $\mathfrak{X}=\mathfrak{X}^{\prime}$. 
The relation " $\preceq$ ” is reflexive and transitive. Moreover, Proposition 1 and Corollary 11 imply that it is also antisymmetric. Therefore " $\preceq$ " is a partial ordering and a performance process is minimal if and only if it is a minimal element relative to this ordering.

Remark. A random variable is usually understood as an equivalence class of measurable mappings which are equal with probability one. Consequently, its values are determined only up to $\mathbf{P}$-negligible set (i.e., a set of $\mathbf{P}$ measure zero). From this point of view, we should require that $Y^{(1)}(\omega) \leq Y^{(2)}(\omega)$ for almost all (instead of all) $\omega \in \Omega$ in Definition 1. Instead, we have taken a slightly different, but equivalent, route, regarding each random variable in our stochastic models as a representative from the coresponding equivalence class (arbitrarily chosen, but fixed thereafter), with well-defined values for each $\omega \in \Omega$. This allows us to make pathwise comparisons for every (instead of almost every) future realization of the stochastic dynamics, but it does not notably alter the results obtained. Indeed, a stochastic model of our network is defined by a countable number of random variables (the initial queue lengths, interarrival and transmission times), so changing their representatives results in a modification of the underlying system only on a $\mathbf{P}$-negligible set.

For given stochastic primitives we have, in general, multiple minimal elements. For instance, minimality of each network with resource sharing and fixed priorities of routes, in which flows on any given route are processed according to SRPT, may be established. For networks with shared resources, minimality assures that the system transmits flows along every route in the SRPT order, see Theorem 2 , to follow. Furthermore, a minimal network is necessarily as efficient (i.e., non-idle) as it can be, given the network topology, the stochastic primitives and the prescribed algorithm for bandwidth allocation between the routes. For a formal justification of this claim, see the remainder of the proof of Theorem 2, where it is demonstrated that a service protocol underutilizing all the resources on any active route cannot be minimal. The theorems presented below are the main results of this article.

Theorem 1. The vector $\mathfrak{X}$ of performance processes defined by (11), corresponding to a resource sharing network operating under the SRPT discipline described in Section 3.3, is minimal.

Proof. Let us fix an arbitrary $\omega \in \Omega$. In the following argument, all random quantities are evaluated at this $\omega$. We argue by contradiction. Suppose that the process $\mathfrak{X}(\omega)$ is not minimal and let a process $\mathfrak{X}^{\prime}=\left(Z^{\prime}, D^{\prime}, T^{\prime}, Y^{\prime}\right)$ be such that $\mathfrak{X}^{\prime} \preceq \mathfrak{X}$, but

$$
\begin{aligned}
\mathfrak{X}^{\prime} & \neq \mathfrak{X} . \text { Let } Q^{\prime}(t)=\lim _{s \rightarrow \infty} Z^{\prime}(t, s), t \geq 0, \text { and let } \\
t_{0} & =\sup \left\{\tilde{t} \geq 0: Y^{\prime}(t, \cdot)=Y(t, \cdot) \forall t \in[0, \tilde{t}]\right\} . \text { (7) }
\end{aligned}
$$

By assumption, we have $Y^{\prime}(0, \cdot)=Y(0, \cdot)=0$, so the set on the right-hand side of $(77)$ is not empty. However, Proposition 1, Corollary 1 and the relation $\mathfrak{X}^{\prime} \neq \mathfrak{X}$ imply that $t_{0}<\infty$. By $(5)$ and the fact that $T(\cdot, s), T^{\prime}(\cdot, s)$ are nondecreasing for each $s$, the latter functions are Lipschitz continuous. Thus, by (4), $Y(\cdot, s), Y^{\prime}(\cdot, s)$ are Lipschitz for each $s$ and hence $Y^{\prime}(t, \cdot)=Y(t, \cdot)$ for all $0 \leq t \leq t_{0}$. Therefore, by (4), Proposition 1 and Corollary 1 , we have

$$
\mathfrak{X}(t, \cdot)=\mathfrak{X}^{\prime}(t, \cdot), \quad 0 \leq t \leq t_{0} .
$$

Put

$$
\begin{gathered}
t_{1}=\inf \left\{t>t_{0}: \triangle N(t) \neq 0 \text { or } \triangle Q(t) \neq 0\right. \\
\text { or } \left.\triangle Q^{\prime}(t) \neq 0\right\},
\end{gathered}
$$

and let $t \in\left[t_{0}, t_{1}\right)$. In what follows, we use the notation from Section 3.3.

First, let us suppose that $Q\left(t_{0}\right) \neq 0$. By the definition of the SRPT discipline, the $k_{0}$-th flow from route $i_{0}$ is processed in the time slot $\left[t_{0}, t_{1}\right)$ by the SRPT system. We consider three cases.

1. For $t \in\left[t_{0}, t_{1}\right)$ and $s \geq w_{i_{0}, k_{0}}\left(t_{0}\right)$, by the relation $\mathfrak{X}^{\prime} \preceq \mathfrak{X},(8)$ and monotonicity of the process $Y^{\prime}$, we have

$$
\begin{aligned}
Y_{i_{0}}^{\prime}(t, s) & \leq Y_{i_{0}}(t, s)=Y_{i_{0}}\left(t_{0}, s\right)=Y_{i_{0}}^{\prime}\left(t_{0}, s\right) \\
& \leq Y_{i_{0}}^{\prime}(t, s) .
\end{aligned}
$$

Hence, for $t, s$ as above,

$$
Y_{i_{0}}^{\prime}\left(t_{0}, s\right)=Y_{i_{0}}^{\prime}(t, s)=Y_{i_{0}}(t, s)=Y_{i_{0}}\left(t_{0}, s\right) .
$$

2. For $t \in\left[t_{0}, t_{1}\right)$ and $s \leq w_{i_{0}, k_{0}}\left(t_{0}\right)-\left(t_{1}-t_{0}\right)$, we have $s<w_{i_{0}, k_{0}}\left(t_{0}\right)-\left(t-t_{0}\right)=w_{i_{0}, k_{0}}(t)$ and hence, by the definition of $i_{0}, k_{0}, Z_{i_{0}}(t, s)=0$. In particular, $Z_{i_{0}}\left(t_{0}, s\right)=0$. By (8),

$$
Z_{i_{0}}^{\prime}\left(t_{0}, s\right)=0 .
$$

The equations (11), (21) and (8)-(9) imply that $Z_{i_{0}}^{\prime}(t, s)=0$ for $t \in\left[t_{0}, t_{1}\right)$, because there are no new arrivals or service completions in the system $\mathfrak{X}^{\prime}$ in the time interval $\left(t_{0}, t_{1}\right)$ and $P_{i}^{\prime}(t, s)=0\left(s<w_{i_{0}, k_{0}}(t)\right.$, so it would be impossible to lower the residual processing time of a flow to $s$ ). Together with (6), this implies that

$$
T_{i_{0}}(t, s)-T_{i_{0}}\left(t_{0}, s\right)=T_{i_{0}}^{\prime}(t, s)-T_{i_{0}}^{\prime}\left(t_{0}, s\right)=0
$$

for $t \in\left[t_{0}, t_{1}\right)$, so for such $t$, by (4),

$Y_{i_{0}}(t, s)-Y_{i_{0}}\left(t_{0}, s\right)=Y_{i_{0}}^{\prime}(t, s)-Y_{i_{0}}^{\prime}\left(t_{0}, s\right)=t-t_{0}$. 
But $Y_{i_{0}}\left(t_{0}, s\right)=Y_{i_{0}}^{\prime}\left(t_{0}, s\right)$ by $(8)$, so $Y_{i_{0}}(t, s)=$ $Y_{i_{0}}^{\prime}(t, s)$.

3. Finally, let $t \in\left[t_{0}, t_{1}\right)$ and $w_{i_{0}, k_{0}}\left(t_{0}\right)-\left(t_{1}-\right.$ $\left.t_{0}\right)<s<w_{i_{0}, k_{0}}\left(t_{0}\right)$. For $t \in\left[t_{0}, t_{0}+w_{i_{0}, k_{0}}\left(t_{0}\right)-s\right)$, we have $w_{i_{0}, k_{0}}(t)>s$. Reasoning similarly as in case 2 (with $t_{1}:=t_{0}+w_{i_{0}, k_{0}}\left(t_{0}\right)-s$ ), we obtain that $Y_{i_{0}}(t, s)=Y_{i_{0}}^{\prime}(t, s)$ for $t \in\left[t_{0}, t_{0}+w_{i_{0}, k_{0}}\left(t_{0}\right)-s\right)$. By continuity, $Y_{i_{0}}\left(t_{0}+w_{i_{0}, k_{0}}\left(t_{0}\right)-s, s\right)=Y_{i_{0}}^{\prime}\left(t_{0}+\right.$ $\left.w_{i_{0}, k_{0}}\left(t_{0}\right)-s, s\right)$. For $t \in\left(t_{0}+w_{i_{0}, k_{0}}\left(t_{0}\right)-s, t_{1}\right)$, we have $w_{i_{0}, k_{0}}(t)<s$. Reasoning similarly as in case 1 (for $t \in\left[t_{0}+w_{i_{0}, k_{0}}\left(t_{0}\right)-s, t_{1}\right)$ ), we have $Y_{i_{0}}^{\prime}(t, s)=Y_{i_{0}}(t, s)$. Therefore $Y_{i_{0}}^{\prime}(t, s)=Y_{i_{0}}(t, s)$ for $t \in\left[t_{0}, t_{1}\right)$.

We have demonstrated that $Y_{i_{0}}^{\prime}(t, s)=Y_{i_{0}}(t, s)$ for all $t \in\left[t_{0}, t_{1}\right), s \geq 0$. get

Proceeding similarly, for $\tilde{\mathbf{I}}:=\left\{i_{0}, \ldots, i_{n-1}\right\}$, we

$$
Y_{i}^{\prime}(t, s)=Y_{i}(t, s), \quad t \in\left[t_{0}, t_{1}\right), \quad s \geq 0, \quad i \in \tilde{\mathbf{I}} .
$$

If $\tilde{\mathbf{I}}=\mathbf{I}$, we have obtained

$$
Y^{\prime}(t, \cdot)=Y(t, \cdot), \quad t \in\left[0, t_{1}\right) .
$$

Otherwise, let $i \in \mathbf{I} \backslash \tilde{\mathbf{I}}$. By the definition of the service discipline in the SRPT resource sharing network, at any time $t \in\left[t_{0}, t_{1}\right)$ no flow on route $i$ is being transmitted. This may be either because of the equality $Q_{i}(t)=0$ on $\left[t_{0}, t_{1}\right)$, or because $i \notin \mathbf{I}_{n}$. In the first case, using (8), we get $Q_{i}^{\prime}\left(t_{0}\right)=Q_{i}\left(t_{0}\right)=0$. This, together with (9), implies that $Q_{i}^{\prime}(t)=0$ for each $t \in\left[t_{0}, t_{1}\right)$. Hence, by (6), for any $s \geq 0$, we have (12) with $i$ instead of $i_{0}$, implying

$$
Y_{i}^{\prime}(t, s)=Y_{i}(t, s), \quad t \in\left[t_{0}, t_{1}\right), s \geq 0,
$$

by the same argument, as the one following (12). If $i \notin \mathbf{I}_{n}$, we have $\mathcal{R}(i) \cap \mathcal{R}\left(i_{m}\right) \neq \emptyset$ for some $i_{m} \in \tilde{\mathbf{I}}$. By the description of the SRPT discipline, for $t \in\left[t_{0}, t_{1}\right)$ and $s \geq w_{i_{m}, k_{m}}\left(t_{0}\right)$, we have $T_{i_{m}}(t, s)-T_{i_{m}}\left(t_{0}, s\right)=t-t_{0}$. Thus, by ( (4) and (13), $T_{i_{m}}^{\prime}(t, s)-T_{i_{m}}^{\prime}\left(t_{0}, s\right)=t-t_{0}$, so by (5) with $j \in \mathcal{R}(i) \cap \mathcal{R}\left(i_{m}\right)$ and monotonicity of $T$, $T^{\prime}$, we have (12) with $i$ instead of $i_{0}$. The increment $T_{i}^{\prime}(t, \cdot)-T_{i}^{\prime}\left(t_{0}, \cdot\right)$ is nondecreasing and nonnegative, and hence validity of (12), with $i$ instead of $i_{0}$, for $s \geq w_{i_{m}, k_{m}}\left(t_{0}\right)$, implies its validity also for $s<w_{i_{n}}, k_{m}\left(t_{0}\right)$. Therefore, by (4) and (8), the relation (15) follows. Consequently, in each case, under the assumption $Q\left(t_{0}\right) \neq 0$, (14) holds, contradicting (77) and (9).

It remains to consider the event in which $Q\left(t_{0}\right)=$ 0 . In this situation, for every $i \in \mathbf{I}$ we can argue as in the case of $i \in \mathbf{I} \backslash \tilde{\mathbf{I}}$ and $Q_{i}(t)=0$ for $t \in\left[t_{0}, t_{1}\right)$ analyzed above.
Our next theorem may be regarded as a partial converse to Theorem 1 .

Theorem 2. Suppose that the process $\mathfrak{X}$ modelling the performance of a network with resource sharing is minimal. Then the flows on every route in this network are prioritized by the SRPT policy.

Proof. Suppose, to the converse, that for some $\omega \in \Omega$ and $\vec{i} \in \mathbf{I}$, the scheduling discipline on the route $\bar{i}$ of the system modelled by the path $\mathfrak{X}(\omega)$ (called system $\mathfrak{X}$ for simplicity) differs from the SRPT policy. In what follows, all random quantities are evaluated at this $\omega$. Define $t_{0}$ as the first time at which task prioritizing on route $\bar{i}$ in the system $\mathfrak{X}$ does not coincide with the SRPT protocol. Deviations from the SRPT order may have three reasons. In the first case, the system $\mathfrak{X}$ may transmit a flow with the remaining transfer time greater than $C_{\bar{i}}\left(t_{0}\right)$. In the second one, there may be (at least) two flows on route $\bar{i}$, say $k$ and $p$, with residual transmission times equal to $C_{\bar{i}}\left(t_{0}\right)$ at time $t_{0}$ such that both of them are transmitted by $\mathfrak{X}$ immediately after $t_{0}$. Finally, it may be the case that $\mathfrak{X}$ uses a service rate lower than the highest available one, although there are flows ready for transmission on route $\bar{i}$ and there is unused capacity at all resources $j \in \mathcal{R}(\bar{i})$. In each of the above cases, we shall find a protocol $\pi^{\prime}$, being a suitable modification of the discipline $\pi$ from $\mathfrak{X}$, such that the resulting trajectory $\mathfrak{X}^{\prime}=\mathfrak{X}^{\prime}(\omega)$ has

$$
T^{\prime}(\omega) \geq T(\omega), \quad T^{\prime}(\omega) \neq T(\omega) .
$$

(We do not change any trajectory $\mathfrak{X}(\tilde{\omega}), \tilde{\omega} \neq \omega$, so $\mathfrak{X}^{\prime}(\tilde{\omega})=\mathfrak{X}(\tilde{\omega})$ for $\tilde{\omega} \neq \omega$ by construction.) Once this task is accomplished, we will get a contradiction with the assumed minimality of $\mathfrak{X}$. In the following argument, for $t \geq 0, i \in \mathbf{I}$ and $k \leq A_{i}(t)$, we will denote the residual transmission time of flow $k$ on route $i$ at time $t$ in the system $\mathfrak{X}^{\prime}$ by $w_{i, k}^{\prime}(t)$.

By definition, the mappings $T_{i}(\cdot, s), i \in \mathbf{I}, s \geq 0$, are nondecreasing. Thus, by (5), they are actually Lipschitz, so absolutely continuous and consequently differentiable almost everywhere (a.e.) with respect to the one-dimensional Lebesgue measure (see, e.g., [20]). Similarly, the residual job service times are Lipschitz, and thus differentiable a.e. with respect to the Lebesgue measure. Note that

$$
\frac{\partial}{\partial t} T_{i}(t, s)=-\sum_{l: 0<w_{i, l}(t) \leq s} \frac{d}{d t} w_{i, l}(t) \quad \text { a.e. }
$$

where the derivative on the left-hand side of the equality is the right derivative of $T_{\bar{i}}(t, s)$ with respect to time. Clearly, the above statements are true for the modified system as well. 
Suppose that $k$ is a flow on route $\bar{i}$ with residual transmission time $C_{\bar{i}}\left(t_{0}\right)$ at time $t_{0}$. The transmission protocol $\pi^{\prime}$ coincides with $\pi$ for each route $i \neq \bar{i}$, and also for route $\bar{i}$, except as described in the following paragraph.

In the first case, denote by $p$ a flow on route $\bar{i}$, with residual transmission time at time $t_{0}$ greater than $C_{\bar{i}}\left(t_{0}\right)$, which is being served at time $t_{0}$ under the protocol $\pi$. Starting from time $t_{0}$, when $\pi$ works on the flow $p, \pi^{\prime}$ transfers the flow $k$, with the same rate, until the completion time $t_{1}$ of the latter transfer. In particular,

$$
w_{i, k}^{\prime}\left(t_{1}\right)=0 .
$$

From time $t_{1}, \pi^{\prime}$ transfers the flow $p$ when $\pi$ transmits $k$, with the same rate. Let $t_{2}=\sup \left\{t \geq t_{0}\right.$ : $\left.w_{i, k}(t)>0\right\}$ be the time at which the system $\mathfrak{X}$ finishes transferring the flow indexed by $k$ on route $\bar{i}$. We will continue the proof under the additional assumption that $t_{1}, t_{2}<\infty$; the proof for the opposite case is an easy modification of the argument given below. By definition,

$$
w_{\bar{i}, k}\left(t_{2}\right)=0 .
$$

For $t \in\left[t_{0}, t_{1}\right)$, we have

$\frac{d}{d t} w_{\bar{i}, k}^{\prime}(t)=\frac{d}{d t} w_{\bar{i}, k}(t)+\frac{d}{d t} w_{\bar{i}, p}(t), \quad \frac{d}{d t} w_{\bar{i}, p}^{\prime}(t)=0$,

a.e., while for $t \in\left[t_{1}, t_{2}\right)$ we have a.e.

$w_{\bar{i}, k}^{\prime}(t)=0, \quad \frac{d}{d t} w_{\bar{i}, p}^{\prime}(t)=\frac{d}{d t} w_{\bar{i}, k}(t)+\frac{d}{d t} w_{\bar{i}, p}(t)$.

All other residual transmission times are equal in both systems.

Clearly, for $t \geq t_{0}$, we have

$$
w_{i, k}^{\prime}(t) \leq w_{\bar{i}, k}(t),
$$

with strict inequality for $t \in\left(t_{0}, t_{2}\right)$. Furthermore, by (20)-(21) and the inequality $w_{i, k}^{\prime}\left(t_{0}\right)=w_{i, k}\left(t_{0}\right)<$ $w_{\bar{i}, p}\left(t_{0}\right)$, for $t \geq t_{0}$,

$$
w_{\bar{i}, k}^{\prime}(t) \leq w_{i, p}(t),
$$

with strict inequality before the time of service completion of the flow $p$ in the system $\mathfrak{X}$.

We claim that for all $t \geq t_{0}$,

$$
w_{\bar{i}, p}^{\prime}(t) \geq w_{\bar{i}, p}(t) .
$$

This is obvious for $t \in\left[t_{0}, t_{1}\right]$. Integrating the first identity in (20) on $\left[t_{0}, t_{1}\right]$ and using (18), we get

$$
\begin{aligned}
w_{\bar{i}, k}\left(t_{0}\right) & =w_{\bar{i}, k}^{\prime}\left(t_{0}\right)= \\
& =w_{\bar{i}, k}\left(t_{0}\right)-w_{\bar{i}, k}\left(t_{1}\right)+w_{\bar{i}, p}\left(t_{0}\right)-w_{\bar{i}, p}\left(t_{1}\right),
\end{aligned}
$$

and hence

$$
w_{\bar{i}, k}\left(t_{1}\right)=w_{\bar{i}, p}\left(t_{0}\right)-w_{\bar{i}, p}\left(t_{1}\right) .
$$

Integrating the second identity in (21) on $\left[t_{1}, t_{2}\right]$ and using (19), (25), we get

$$
\begin{aligned}
w_{\bar{i}, p}^{\prime}\left(t_{2}\right) & =w_{\bar{i}, p}^{\prime}\left(t_{1}\right)-w_{\bar{i}, k}\left(t_{1}\right)+w_{\bar{i}, p}\left(t_{2}\right)-w_{\bar{i}, p}\left(t_{1}\right) \\
& =w_{\bar{i}, p}^{\prime}\left(t_{1}\right)-w_{\bar{i}, p}\left(t_{0}\right)+w_{\bar{i}, p}\left(t_{2}\right)=w_{\bar{i}, p}\left(t_{2}\right),
\end{aligned}
$$

because $w_{\bar{i}, p}^{\prime}\left(t_{1}\right)=w_{\bar{i}, p}^{\prime}\left(t_{0}\right)=w_{\bar{i}, p}\left(t_{0}\right)$ by the second identity in (20) and the definition of $\mathfrak{X}^{\prime}$. The second identity in (21) implies that for $t \in\left[t_{1}, t_{2}\right]$ we have $\frac{d}{d t} w_{\bar{i}, p}^{\prime}(t) \leq \frac{d}{d t} w_{i, p}(t)$ a.e., which, together with (26), implies (24) for $t \in\left[t_{1}, t_{2}\right]$. Finally, (26) and the definition of $\mathfrak{X}^{\prime}$ imply that

$$
w_{i, p}^{\prime}(t)=w_{i, p}(t), \quad t \geq t_{2},
$$

and the proof of (24) is complete.

By the definition of $\pi^{\prime}$, for $0 \leq t \leq t_{0}$ we have $T_{\bar{i}}^{\prime}(t, \cdot)=T_{\bar{i}}(t, \cdot)$. By (17), (20) and (22)-(23), for $t \in$ $\left[t_{0}, t_{1}\right)$, we have $\frac{\partial}{\partial t} T_{\bar{i}}^{\prime}(t, s) \geq \frac{\partial}{\partial t} T_{\bar{i}}(t, s)$. Integrating this inequality with respect to $t$ from $t_{0}$ to $t$, we get

$$
T_{\bar{i}}^{\prime}(t, s) \geq T_{\bar{i}}(t, s), \quad t \in\left[t_{0}, t_{1}\right], \quad s \geq 0 .
$$

Moreover, for $t \in\left(t_{0}, t_{1}\right)$ and $s=w_{\bar{i}, k}^{\prime}(t)$, the inequality in (28) is strict due to strict inequalities in (22), (23).

For $t, s \geq 0$, let $\tilde{T}_{\bar{i}}(t, s)$ denote the cumulative transmission time of all the flows on route $\bar{i}$ except for $k, p$ by time $t$ with residual transmission times (evaluated at the times of their processing) less than or equal to $s$. Note that this quantity is the same in both systems by the definition of $\mathfrak{X}^{\prime}$. For any $t, s \geq 0$, we have

$$
\begin{aligned}
T_{\bar{i}}(t, s) & =\tilde{T}_{\bar{i}}(t, s)+\left(w_{\bar{i}, k}^{0} \wedge s\right) \\
& -\left(w_{\bar{i}, k}(t) \wedge s\right)+\left(w_{\bar{i}, p}^{0} \wedge s\right)-\left(w_{\bar{i}, p}(t) \wedge s\right), \\
T_{\bar{i}}^{\prime}(t, s) & =\tilde{T}_{\bar{i}}(t, s)+\left(w_{\bar{i}, k}^{0} \wedge s\right) \\
& -\left(w_{\bar{i}, k}^{\prime}(t) \wedge s\right)+\left(w_{\bar{i}, p}^{0} \wedge s\right)-\left(w_{\bar{i}, p}^{\prime}(t) \wedge s\right),
\end{aligned}
$$

so the inequality in (16) is equivalent to the relation $\left(w_{\bar{i}, k}(t) \wedge s\right)+\left(w_{\bar{i}, p}(t) \wedge s\right) \geq\left(w_{\bar{i}, k}^{\prime}(t) \wedge s\right)+\left(w_{i, p}^{\prime}(t) \wedge s\right)$, taking the form

$$
\left(w_{\bar{i}, k}(t) \wedge s\right)+\left(w_{\bar{i}, p}(t) \wedge s\right) \geq w_{\bar{i}, p}^{\prime}(t) \wedge s
$$

for $t \geq t_{1}$, due to $(18)$. 
If either $w_{\bar{i}, k}(t) \geq s$ or $w_{\bar{i}, p}(t) \geq s$, then (29) clearly holds. In the opposite case, (29) is implied by the equality

$$
w_{\bar{i}, k}(t)+w_{\bar{i}, p}(t)=w_{\bar{i}, p}^{\prime}(t) .
$$

For $t=t_{1}$, (30) holds by (25), the second equation in (20) and the equality $w_{\bar{i}, p}\left(t_{0}\right)=w_{\bar{i}, p}^{\prime}\left(t_{0}\right)$. This, together with the second equation in (21) , yields (30) for $t \in\left[t_{1}, t_{2}\right]$. Finally, by (19), for $t \geq t_{2}$, (30) boils down to (27). We have proved (29) for $t \geq t_{1}$, so the proof of (16) in the case under consideration is complete.

The proof of (16) in the second case is almost the same as in the previous one, the only notable difference is that now we have $w_{\bar{i}, k}^{\prime}\left(t_{0}\right)=w_{\bar{i}, k}\left(t_{0}\right)=$ $w_{\bar{i}, p}\left(t_{0}\right)$ instead of the strict inequality above (23)

It remains to consider the third case. Here, starting from time $t_{0}$, when we have unused capacity at every resource $j \in \mathcal{R}(\bar{i})$ under $\pi$, the protocol $\pi^{\prime}$, in addition to executing the transfers of $\pi$, transfers the flow $k$, with the highest possible rate, until the ending time $t_{1}$ of this transfer. Starting from the time $t_{1}$, whenever $\pi$ transfers the flow $k$, the protocol $\pi^{\prime}$ lets its transfer rate on route $\bar{i}$ go unused (i.e., idles).

We will proceed with a more formal description of $\pi^{\prime}$. For $j \in \mathbf{J}$ and $t, s \geq 0$, let

$$
T^{j}(t, s)=\sum_{i \in \mathcal{F}(j)} T_{i}(t, s)
$$

be the cumulative transfer time devoted to flows with residual transmission times less than and equal to $s$ by the resource $j$ on the time horizon $[0, t]$ and let

$$
\bar{T}_{j}(t)=\lim _{s \rightarrow \infty} T^{j}(t, s)
$$

be the total transfer time of $j$ on $[0, t]$. By definition, the mappings $\bar{T}_{j}$ are nondecreasing. Thus, by (5), they are also Lipschitz, and hence differentiable a.e.. For $t \geq t_{0}$, let $r(t)$ be the spare capacity of the system $\mathfrak{X}$ for route $\bar{i}$ in $\left[t_{0}, t\right]$, so that $r\left(t_{0}\right)=0$ and

$$
\frac{d r(t)}{d t}=\mathbb{I}_{\left[Q_{\bar{i}}(t)>0\right]}\left(1-\max _{j \in \mathcal{R}(\bar{i})} \frac{d \bar{T}_{j}(t)}{d t}\right) \text { a.e. on }\left[t_{0}, \infty\right) \text {. }
$$

By the assumption of this case, we have $r(t)>0$ for every $t>t_{0}$. By the definition of the policy $\pi^{\prime}$, for $t \geq t_{0}$, we have $w_{\bar{i}, k}^{\prime}(t)=\left(w_{\bar{i}, k}(t)-r(t)\right)^{+}$, so

$w_{\bar{i}, k}^{\prime}(t) \leq w_{\bar{i}, k}(t), \quad t \geq t_{0}$,

$w_{i, k}^{\prime}(t)<w_{\bar{i}, k}(t), t_{0}<t<\inf \left\{t \geq t_{0}: w_{\bar{i}, k}(t)=0\right\}$.

For $t, s \geq 0$, let $\tilde{T}_{\bar{i}}(t, s)$ denote the cumulative transmission time of all the flows on route $\bar{i}$ except for $k$ by time $t$ with residual transmission times (evaluated at the times of their processing) less than or equal to $s$. Note that this quantity is the same in both systems by the definition of $\pi^{\prime}$. As in the first case, if $0 \leq t \leq t_{0}$ then $T_{\bar{i}}^{\prime}(t, \cdot)=T_{\bar{i}}(t, \cdot)$. For $t>t_{0}$, we have

$$
\begin{aligned}
& T_{\bar{i}}(t, s)=\tilde{T}_{\bar{i}}(t, s)+\left(w_{\bar{i}, k}^{0} \wedge s\right)-\left(w_{\bar{i}, k}(t) \wedge s\right), \\
& T_{\bar{i}}^{\prime}(t, s)=\tilde{T}_{\bar{i}}(t, s)+\left(w_{\bar{i}, k}^{0} \wedge s\right)-\left(w_{\bar{i}, k}^{\prime}(t) \wedge s\right),
\end{aligned}
$$

so (32)-(33) imply that $T_{\bar{i}}^{\prime} \geq T_{\bar{i}}, T_{\bar{i}}^{\prime} \neq T_{\bar{i}}$ and (16) holds.

An immediate consequence of Theorems 1 and 2 for the case of $I=J=1$ is

Corollary 2. The performance process corresponding to a one-server queue with a single customer class is minimal if and only if the underlying queue discipline is SRPT.

\section{Conclusion}

In this paper, we investigate general resource sharing networks with arbitrary topology, making no distributional assumptions on the underlying interarrival or service times. We introduce a new idleness-based optimality criterion, suitable for performance analysis of job size-based policies for such networks, which is particularly sensitive to the service of small tasks. We then use it to make pathwise comparisons of the outcomes of implementing different transmission schemes for a given network. It turns out that the "greedy" SRPT network protocol is minimal with respect to our criterion and, moreover, any minimizing policy necessarily applies SRPT as an intra-route discipline.

Our study appears to be the first one addressing the implementation of the SRPT protocol to networks with shared resources in this generality. It also identifies a natural optimality criterion characterizing, to some extent, the SRPT policy in this context. It may be somewhat surprising that any strictly subcritical SRPT resource sharing network is minimal, as defined in this paper, although it may actually turn out to be unstable [12]. This indicates that these two performance criteria are different in nature.

A natural extension of the results presented here will be an investigation of a stronger, more refined notion of edge minimality, analogous to the one developed in [16] for real-time networks. Its usage as a performance criterion would enforce assigning transmission priorities to routes, as well as intra-route flow priorities, consistently with the SRPT protocol. It would also be useful to generalize our findings to networks with different resource capacities. Both these directions should be investigated in future work. 


\section{References:}

[1] L. E. Schrage, L. W. Miller, The queue M/G/1 with the shortest remaining processing time discipline. Operations Research 14 (1966), 670-684.

[2] L. E. Schrage, A proof of the optimality of the shortest remaining processing time discipline. Operations Research 16 (1968), 687-690.

[3] F. Schreiber, Properties and applications of the optimal queueing strategy SRPT: a survey. Archiv für Elektronik und Übertragungstechnik, 47 (1993), 372-378.

[4] A. Wierman, M. Harchol-Balter, Classifying scheduling policies with respect to unfairness in an M/G/1. In Proceedings of the 2003 ACM SIGMETRICS International Conference on Measurement and Modeling of Computer Systems, 238249, 2003.

[5] R. Núñez-Queija, Queues with equally heavy sojourn time and service requirement distributions. Annals of Operations Research 113 (2002), 101117.

[6] M. Nuyens, B. Zwart, A large deviations analysis of the GI/GI/1 SRPT queue. Queueing Systems 54 (2006), 85-97.

[7] A. V. Pavlov, A system with Schrage servicing discipline in the case of a high load. Engrg. Cybernetics 21 (1984), 114-121; translated from Izv. Akad. Nauk SSSR Tekhn. Kibernet. 6 (1983), 5966 (Russian).

[8] A. V. Pechinkin, Heavy traffic in a system with a discipline of priority servicing for the job with the shortest remaining length with interruption (Russian). Math. Issled. No. 89, Veroyatn. Anal. 97 (1986), 85-93.

[9] S. Banerjee, A. Budhiraja, A. L. Puha, Heavy traffic scaling limits for shortest remaining processing time queues with heavy tailed processing time distributions. arXiv: 2003.03655v1 (2020).

[10] L. Massoulié, J. Roberts (1999) Bandwidth sharing: objectives and algorithms. In Proceedings of the INFOCOM '99, Eighteenth Annual Joint Conference of the IEEE Computer and Communications Societies, vol 3, pp 1395-1403, IEEE.

[11] L. Massoulié, J. Roberts, Bandwidth sharing and admission control for elastic traffic. Telecommunication Systems 15 (2000), 185-201.

[12] M. Verloop, S. Borst and R. Núñez-Queija, Stability of size-based scheduling disciplines in resource-sharing networks. Performance Evaluation 62 (2005), 247-262.
[13] S. Aalto, U. Ayesta, SRPT applied to bandwidth sharing networks, Ann. Oper. Res. 170 (2009), 319.

[14] T. Chojecki, Ł. Kruk, Instability of SRPT, SERPT and SJF queueing networks, working paper, Maria Curie-Skłodowska University, Lublin, Poland (2020)

[15] Ł. Kruk, Minimality of EDF networks with resource sharing. Math. Meth. Oper. Res. 84 (2016), 259-283.

[16] Ł. Kruk, Edge minimality of EDF resource sharing networks. Math. Meth. Oper. Res. 86 (2017), 331-366.

[17] A. Espinal, R. Estrada, C. Monsalve, Modelling TCP/IP traffic of a convergent campus wireless network, International Journal of Circuits, Systems and Signal Processing 13 (2019), 611-616.

[18] J. Zhou, A path-server traffic scheduling algorithm for wireless network load based on SDN, International Journal of Circuits, Systems and Signal Processing 14 (2020), 855-862.

[19] Yu. V. Prohorov, Convergence of random processes and limit theorems in probability theory. Theory of Probability and its Applications 1 (1956), 157-214.

[20] R. L. Wheeden, A. Zygmund. Measure and Integral: An Introduction to Real Analysis. 2nd Edition, Chapman and Hall/CRC Press, Boca Raton, FL, 2015.

\section{Contribution of individual authors to the creation of a scientific article (ghostwriting policy)}

The second author has suggested this research project, as well as the corresponding methodology.

Both authors were actively involved in the investigation and the formal analysis, writing the original draft of this paper, its revised version and visualization of the work presented here.

\section{Creative Commons Attribution License 4.0 (Attribution 4.0 International, CC BY 4.0)}

This article is published under the terms of the Creative Commons Attribution License 4.0 https://creativecommons.org/licenses/by/4.0/deed.en US 